\title{
Einführung in die Thematik: Zwischen Aushandlung und Direktive - Kulturpolitische Steuerungsmodelle und Theater
}

\section{Gerald Siegmund}

\section{Schlüsselwörter}

Kulturpolitik • Steuerungsmodelle • Zielvereinbarungen • Gesellschaftliche Leitbilder • Organisationsformen des Theaters

„Nie hat Politik so permanent und mächtig in den aktuellen Prozess des Kunstund Kulturschaffens hineingewirkt wie im Augenblick“, schreibt Thomas Oberender, Intendant der Berliner Festspiele, in einem Beitrag zu einem Sammelband über „kulturpolitische Konzeptionen zur Reform der Darstellenden Künste“ (Oberender 2013, S. 80; Schneider 2013). Folgen wir Oberenders These, so ergibt sich daraus ein latentes Spannungsverhältnis zwischen Politik und den Theatern, deren Freiheit Politik doch gewährleisten soll. Garantiert Artikel 5 des Grundgesetzes die Kunstfreiheit, dürfte sich Kulturpolitik eigentlich gar nicht in den ,Prozess des Kunst- und Kulturschaffens“ einmischen, respektive in diesen hineinwirken. Politik stellt lediglich die rechtlichen und finanziellen Rahmenbedingungen bereit, innerhalb derer Kunstschaffende ,frei“ handeln können. Dennoch gibt es selbstredend Steuerungsmodelle, die das Verhältnis zwischen den Theatern und deren öffentlichen Trägern regeln. Diese folgen nun keineswegs nur formalen Kriterien, sondern transportieren immer schon Inhalte, die die Theater organisatorisch und inhaltlich umsetzen sollen. Wie Politik dies tut, ist die Frage, die in diesem Kapitel verhandelt werden soll. Das Verhältnis zwischen den öffentlichen Trägern und den Theatern wird einerseits durch Zielvereinbarungen bestimmt, die etwa die Anzahl

\footnotetext{
G. Siegmund $(\varangle)$

Institut für Angewandte Theaterwissenschaft, Justus-Liebig-Universität Gießen, Gießen , Deutschland

E-Mail: gerald.siegmund@ theater.uni-giessen.de
} 
der Premieren pro Spielzeit festlegen. Wie das Beispiel des Mitbestimmungsmodells an den Städtischen Bühnen Frankfurt am Main in den 1970er Jahren gezeigt hat, sind diese Vereinbarungen immer auch Spiegel gesellschaftspolitischer Vorstellungen, die sich in politischen Agenden niederschlagen. Wenn dies der Fall ist, betreffen die Steuerungsmodelle immer auch die Werte und die Legitimationsbasis der Institution Theater. Was sollen die Theater umsetzen? Für den Frankfurter Kulturdezernenten Hilmar Hoffmann war dies in den 1970er Jahren klar. Theater sollen Teilhabe auch der unteren Gesellschaftsschichten garantieren und zur individuellen wie gesellschaftlichen Emanzipation durch kritisches Denken, die Schärfung der Wahrnehmung sowie die Ausbildung der Phantasie beitragen (Hoffmann 1979, S. 53). Wie dies im Einzelnen geschieht, bleibt den Theatern überlassen. Damit sind die Steuerungsmodelle auch dem Wandel gesellschaftlicher Leitbilder unterworfen, die nicht allein das Verhältnis von Politik zur Verwaltung von Theatern betrifft. So schlagen sich neue Managementmodelle nicht nur in den Verwaltungsstrukturen nieder. Vielmehr sind sie Ausdruck eines viel weitreichenderen ökonomischen und kulturellen Wertewandels, der sich seit den 1990er Jahren auch in der Etablierung einer Freien Szene abseits traditioneller Stadt- und Staatstheaterstrukturen artikuliert. Individualisierte, projektbasierte Produktionsstrukturen in flexiblen Teams mit wechselnder, immer wieder neu zu beantragender Finanzierung durch verschiedene Geldgeber stehen dabei der Förderung einzelner feststehender Häuser gegenüber, die gleichsam als Zwischeninstanz einzelne Inszenierungen ermöglichen. Thomas Oberender beschreibt diesen institutionellen Wandel, der mit einem Wandel der Steuerungsmodelle und damit der Organisationsform der Theater einhergeht, als eine Entwicklung hin $\mathrm{zu}$ „Patchworkinstitutionen“, die sich durch andere Institutionen (Kulturfonds, Stiftungen, Kurator*innen, Künstler*innengruppen) legitimieren und am Leben erhalten (Oberender 2013, S. 70).

In den Beiträgen dieses Kapitels werden verschiedenen Möglichkeiten der kulturpolitischen Steuerung diskutiert. Johannes Crückeberg und Moritz Steinhauer zeichnen in ihrem Beitrag anhand von Beispielen die historische Entwicklung nach, die zur Veränderung kulturpolitischer Steuerung von einem klassisch bürokratischen über ein manageriales Modell bis hin zur Cultural Governance führte. Julia Glesner beleuchtet in ihrem Beitrag die Funktion der aufsichtsführenden Gremien für einen funktionierenden Theaterbetrieb. Ulrike Hartung widmet sich im Anschluss daran einer nahezu klassischen Möglichkeit, kulturpolitisch Einfluss zu nehmen auf die Programme der Theater: die Intendantenbesetzung. Anhand der Rechtsstreitigkeiten um Adolphe Binders Position als Intendantin des Tanztheaters Wuppertal analysiert sie das Spannungsverhältnis zwischen kulturpolitischer Steuerung de jure und ihrer faktischen Umsetzung in institutioneller Praxis. Dass 
gerade die Einführung eines managerialen Steuerungsmodells und der damit verbundenen Ausgliederung der Theater aus der Verwaltung Anfang der 1990er Jahre für die Theater in Ost- und Westdeutschland eine erhöhte Abhängigkeit von der Politik bedeutet, beleuchten Lara Althoff, Jonas Marggraf und Annette Zimmer in ihrem Beitrag. In einem Vergleich der Rechtsformen der Theater in den ostdeutschen Ländern mit drei Theatern in NRW stellen sie nach der Wiedervereinigung eine Angleichung der kulturpolitischen Steuerung fest.

\section{Literatur}

Hoffmann, Hilmar. 1979. Kultur für Alle. Frankfurt a. M.: S. Fischer.

Oberender, Thomas. 2013. Ein Theater neuen Typs. Kulturpolitische Wege der Infarktbekämpfung. In Theater entwickeln und planen. Kulturpolitische Konzeptionen zur Reform der Darstellenden Künste, Hrsg. Wolfgang Schneider, 69-89. Bielefeld: Transcript.

Schneider, Wolfgang, Hrsg. 2013. Ein Theater neuen Typs. Kulturpolitische Wege der Infarktbekämpfung. In Theater entwickeln und planen. Kulturpolitische Konzeptionen zur Reform der Darstellenden Künste. Bielefeld: Transcript.

Open Access Dieses Kapitel wird unter der Creative Commons Namensnennung 4.0 International Lizenz (http://creativecommons.org/licenses/by/4.0/deed.de) veröffentlicht, welche die Nutzung, Vervielfältigung, Bearbeitung, Verbreitung und Wiedergabe in jeglichem Medium und Format erlaubt, sofern Sie den/die ursprünglichen Autor(en) und die Quelle ordnungsgemäß nennen, einen Link zur Creative Commons Lizenz beifügen und angeben, ob Änderungen vorgenommen wurden.

Die in diesem Kapitel enthaltenen Bilder und sonstiges Drittmaterial unterliegen ebenfalls der genannten Creative Commons Lizenz, sofern sich aus der Abbildungslegende nichts anderes ergibt. Sofern das betreffende Material nicht unter der genannten Creative Commons Lizenz steht und die betreffende Handlung nicht nach gesetzlichen Vorschriften erlaubt ist, ist für die oben aufgeführten Weiterverwendungen des Materials die Einwilligung des jeweiligen Rechteinhabers einzuholen. 